\title{
Strain rate and flame orientation statistics in the near-wall region for turbulent flame-wall interaction
}

\author{
Peipei Zhao ${ }^{\mathrm{a}}$, Lipo Wang ${ }^{\mathrm{a} *}$ and Nilanjan Chakraborty ${ }^{\mathrm{b}}$ \\ ${ }^{a}$ UM-SJTU Joint Institute, Shanghai Jiao Tong University, Shanghai 200240, China \\ ${ }^{b}$ School of Engineering, Newcastle University, Newcastle-Upon-Tyne, UK
}

(Compiled February 12, 2018)

\begin{abstract}
Flame-wall interaction (FWI) in premixed turbulent combustion has been analyzed based on a counter-flow like configuration at the statistically stationary state. For the present configuration, the two FWI sub-zones, i.e the influence zone and the quenching zone, can be quantified from the DNS results. Detailed analysis of the important quantities, such as the flame temperature, flame-wall distance, wall heat flux, flame curvature and dilatation (including the flame normal and tangential strain rates), and some orientation relations between the flame normal and the principal strain rate directions, have been reported, together with the physical explanations. All these statistical results are determined by the relative strengths of the wall heat flux, thermal expansion and the flame-turbulence interaction.
\end{abstract}

Keywords: flame-wall interaction; premixed turbulent combustion; strain rate; wall heat flux

\section{Introduction}

In most combustion devices such as in internal combustion engines the flame-wall interaction (FWI) is inevitable because the combustion process takes place in a confined space. From the consideration of downsizing the combustors, the flame and flow in the vicinity of the wall are very different from the free propagating case. The local flame quenching and heat loss through the boundary as a result of FWI have important implications on the overall efficiency, pollution control and the combustor durability. However, most existing premixed turbulent flame models [1-6] do not include the FWI effects. Therefore, both fundamental understanding of FWI and its modeling are necessary for the development of high-fidelity predictive tools for engineering design of new generation light-weight compact energy-efficient combustors.

Some early results of FWI were obtained from a total quenching analysis of a laminar tube flow [7-11], which is significantly different from most practical FWI events in engineering applications. For the turbulent cases, two existing flow configurations are the head-on quenching case and the side-wall quenching case. Fig. 1 (a) shows the head-on quenching configuration, where the premixed flame propagates toward the cold wall along the wall normal direction. Without mass and energy feeding, the premixed flame continuously consumes the reactant on the wall side and finally approaches the wall till it completely quenches. Besides, the turbulence intensity decays because of energy dissipation and also due to the action of increasing flow strain. Some

*Corresponding author. Email: lipo.wang@sjtu.edu.cn 
important results on FWI have been obtained from such unsteady head-on quenching analysis. For example, the maximum wall heat flux magnitude for both the laminar and turbulent cases and the quenching distance (i.e. the minimum distance $\delta_{Q}$ between the flame and the wall such that flame quenches for $x<\delta_{Q}$ ) can be quantified [12 13]. The flame quenching distance $\delta_{Q}$ and the maximum heat flux magnitude $\left.Q\right|_{w}$ are often expressed in the normalized form as $P_{Q}=\delta_{Q} / \delta_{z}$ and $\left.\tilde{Q}\right|_{w}=\left.Q\right|_{w} / \rho_{u} S_{L} C_{p, u}\left(T_{a d}-T_{u}\right)$ with $\delta_{z}, S_{L}, \rho_{u}, T_{u}, T_{a d}$ and $C_{p, u}$ being the Zeldovich thickness, unstrained laminar flame speed, unburned gas density, unburned gas temperature, adiabatic flame temperature and specific heat of the fresh gas respectively. The Zeldovich thickness is defined as $\delta_{z}=D_{t h} / S_{L}$ where $D_{t h}$ is the unburned gas thermal diffusivity. It has been indicated that the quenching Peclet number is inversely proportional to the maximum wall heat flux magnitude [12 14 15. Poinsot et al.[12] and Lai et al.[13] showed that $P_{Q}$ is about 3, corresponding to a maximum wall heat flux $\left.\tilde{Q}\right|_{w}$ about $0.3 \sim 0.4$. Further investigations suggest that these critical numbers, $P_{Q}$ and $\left.\tilde{Q}\right|_{w}$, will depend on the wall boundary condition [16 18, initial gas temperature [1920] and the pressure [16,20,21]. In addition, the local or even global flame structure under the influence of FWI have also been investigated [12, including the interaction between the vortex and the flame and the dominant role of the vortex pair in FWI. Gruber et al.[22] analyzed the side-wall quenching of a $\mathrm{V}$-shaped turbulent flame in a confined channel. It shows that FWI has strong effects on the flame thickness, and flow structure in the near-wall region. For this case both the quenching Peclet number and the normalized maximum wall heat flux are different from the head-on quenching case. Moreover, in the near-wall region, because of the large wall heat flux, the thin flamelet regime will change to a thickened wrinkled regime.

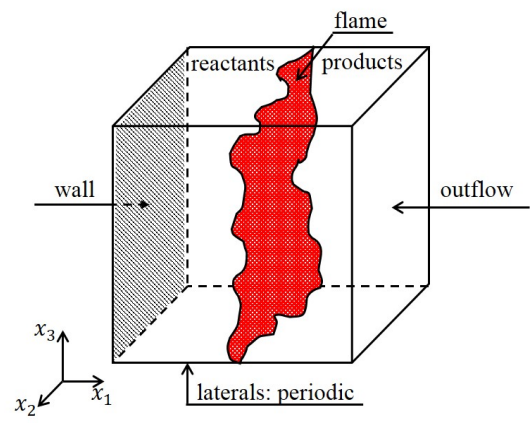

(a) Conventional model

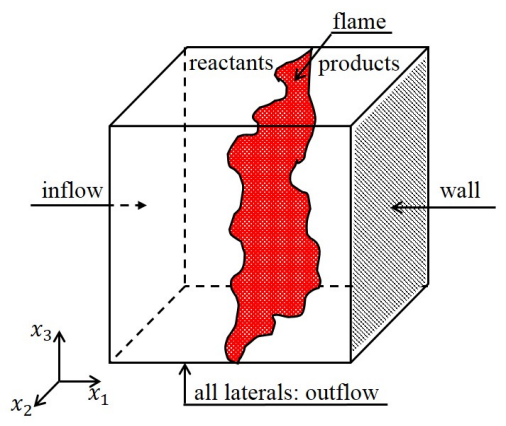

(b) New model

Figure 1. The configuration for FWI.

However, under practical operating conditions the FWI scenario is often quite different from the configurations considered in previous analyses [12 1322]. Because of the mass and energy transportation by the main stream, the turbulent flame flushes continuously to the solid wall in most engineering applications. As shown in Fig. 1 (b), the flow and flame can finally reach statistically stationary state, which closely resembles to the combustion processes in gasoline engines and gas turbines where the flame is convected by large scale eddies to flush over the wall boundary. Furthermore, experimental analysis by Mann et al. 23 revealed differences in flame orientation between transient HOQ and steady-state flame stabilized by a wall. Thus, it can be expected that FWI under statistically stationary state can be different from the evolving cases, 
but this is rarely addressed in the existing literature. Therefore, an alternative FWI configuration is proposed in this work. As schematically shown in Fig. 1(b), the flame is convected by the inflow of fresh reactants and anchored by the solid wall at some location, which physically is determined by the inflow mass flux, i.e. the incoming flow speed, and the wall boundary conditions. The lateral surfaces are set as the outflow boundary. In contrast to Fig. 1 (a), the flame can be statistically stationary in Fig. 11(b) and the reactant/product distribution is different. Roughly the overall energy balance relations can be described by the following one-dimensional relation

$$
Q_{\dot{\omega}}=\rho_{u} u_{1, u} C_{p}\left(T_{b}-T_{u}\right)+Q_{w},
$$

where $\rho$ is the density, $u_{1}$ is the velocity in streamwise direction, $T$ is the temperature, $Q_{\dot{\omega}}$ is the reaction heat release rate, $C_{p}$ is the specific heat, and $Q_{w}$ is roughly the wall heat loss. In the following the subscript ' $u$ ' and ' $b$ ' denote the quantity in the reactant side and product side, respectively. As the flame approaches the wall, the wall heat loss $Q_{w}$ increases and thus the downstream flame temperature $T_{b}$ decreases and the flame eventually quenches once $T_{b}$ is smaller than a critical value. Therefore the wall boundary condition, especially the wall temperature, plays an important role in detailed physical understanding of FWI.

The objective of the present work is to explore the fundamental physics of FWI based on a new configuration shown by Fig. 1 (b). Especially the adiabatic no-slip wall boundary and isothermal no-slip wall boundary cases will be compared to understand the effects of wall boundary condition on FWI. In the following, after a brief description of the direct numerical simulation (DNS), we will focus on the discussion of the results and their analyses, and end up with concluding remarks.

\section{Numerical simulations}

The three-dimensional compressible reacting flow in the configuration shown in Fig. 1(b) is numerically simulated. All the spatial derivatives adopt a 10th order central difference scheme for the internal points while the scheme order decreases gradually to an one-sided 2nd order finite difference approximation at the non-periodic boundary points [24]. An explicit third-order low storage Runge-Kutta scheme [25] is used for the temporal integration. A steady planar laminar flame solution [26] is prescribed as the initial condition for the scalar fields. The chemical kinetics here is simplified as an one-step irreversible ('Reactants $\rightarrow$ Products') mechanism following the Arrhenius law. It has been demonstrated in previous DNS analyses [12 13 15 2729] that premixed flames quench near the cold wall principally due to the heat loss and also due to the strain rate induced by the wall. It is worth noting that the main focus of the present work is on the combustion physics, instead of the chemical pathways analysis. The results from single-step chemistry DNS agree well with the experiment measurements of the quenching distance and the wall heat flux [35 36], especially at the low wall temperature [18. An experimental analysis by Jainski et al. 30] indicated that the near-wall dynamics can be accurately captured by simple chemistry DNS. A recent detailed chemistry based investigation (16 species and 25 reactions by Lai et al. 31 indicated that the most FWI physics can be, at least qualitatively if not quantitatively, captured by simple chemistry simulations with constant thermo-physical properties. Poinsot et al.[32] showed that the use of temperaturedependent thermo-physical properties does not change the qualitative nature of the 
flame-turbulence interaction obtained from constant thermo-physical properties. The same conclusion was drawn for flame-vorticity interaction by Louch and Bray 33. In a recent paper Aspden 34 demonstrated based on 3D detailed chemistry DNS that constant viscosity simulations yielded qualitatively similar picture when compared to the simulation results with variable thermo-physical properties. In this analysis constant thermo-physical properties have been assumed for the purpose of simplicity so that the fundamental physics can be captured. In order to substantiate that the results presented in this paper remain at least qualitatively similar in the presence of temperature-dependent thermo-physical properties, some additional simulations have been carried out, where the Sutherlands law is adopted for the dynamics viscosity as $\mu=\mu_{\text {ref }}\left(T / T_{\text {ref }}\right)^{3 / 2}\left(T_{\text {ref }}+S\right) /(T+S)$ (correspondingly, the thermal conductivity and mass diffusivity are temperature dependent as well), where $S$ is the Sutherland temperature. These simulation results are shown in Appendix and they reveal that the main physical picture remains qualitatively valid but some minor differences were observed in quantitative sense. Subject to the above assumptions, the chemical mechanism reads $\dot{\omega}=B \rho(1-c) \exp \left[-\frac{\beta(1-\tilde{T})}{1-\alpha(1-\tilde{T})}\right]$, where $B$ is the normalised pre-exponential factor, $\alpha=\frac{\tau}{\tau+1}$ with $\tau$ being the heat release parameter. The nondimensionalized temperature $\tilde{T}$ is defined as $\tilde{T}=\frac{T-T_{u}}{T_{a d}-T_{u}}$, and $c=\frac{Y_{R, u}-Y_{R}}{Y_{R, u}-Y_{R, b}}$ is the reaction progress variable, where $Y_{R, u}$ and $Y_{R, b}$ represent the reactant mass fraction in the fresh reactants stream and in the fully burned products, respectively. The reaction progress variable $c$ increases from zero in the fresh reactant side to unity in the burned product side. Other relevant quantities are non-dimensionalized by the corresponding reference values. Specifically, the domain size $L$ and the laminar flame speed $S_{L}$ are chosen as the reference length $l_{r}$ and reference velocity $u_{r}$, respectively; the reference density $\rho_{r}$ and the other reference thermo-physical properties such as specific heat $C_{p, r}$, thermal conductivity $\lambda_{r}$ and mass diffusivity $D_{r}$ are taken to be equal to the corresponding values in the unburned gas. Besides, the specific heats and the thermo-physical properties such as, viscosity $\tilde{\mu}$, thermal conductivity $\tilde{\lambda}$ and the density-weighted mass diffusivity $\tilde{\rho} \tilde{D}$ are assumed to be constant and independent of temperature. In summary, the characteristic flow parameters and the flame parameters are listed in Table. 1. A value of $\tau=\left(T_{a d}-T_{u}\right) / T_{u}=2.3$ is representative of methane-air mixture preheated to $750 \mathrm{~K}$. Please note that similar values of have been used in several previous DNS analyses in the past [37-40].

Table 1. Characteristic flow and flame parameters.

\begin{tabular}{|c|c|c|c|c|c|c|}
\hline $\operatorname{Pr}_{r}$ & $S c_{r}$ & $L e$ & $M a_{r}$ & $\gamma$ & $\tau$ & $\beta$ \\
\hline 0.7 & 0.7 & 1.0 & 0.014 & 1.4 & 2.3 & 6.0 \\
\hline
\end{tabular}

*The Prandtl number $\operatorname{Pr}_{r}=\frac{\mu_{r} C_{p, r}}{\lambda_{r}}$, the Schmidt number $S c_{r}=\frac{\mu_{r}}{\rho_{r} D_{r}}$, the Lewis number $L e=S c / \operatorname{Pr}$, the Mach number $M a_{r}=\frac{u_{r}}{a_{r}}\left(a_{r}=\sqrt{\gamma R_{g} T_{u}}\right)$, the ratio of specific heats $\gamma$, the heat release number $\tau=$ $\frac{T_{a d}-T_{u}}{T_{u}}$, the Zeldovich number $\beta=\frac{T_{a}\left(T_{a d}-T_{u}\right)}{T_{a d}{ }^{2}}\left(T_{a}\right.$ : the activation temperature).

Numerically, the velocity boundary condition on the inflow surface is the combination of the mean part $U_{i}\left(U_{1} / S_{L}=8.0\right.$ and $\left.U_{2}=U_{3}=0\right)$ and the fluctuating part $u_{i}^{\prime}\left(u_{i}^{\prime} / S_{L}=2.0\right)$, which is specified by scanning a plane through an auxiliary homogeneous isotropic turbulent field generated a priori based on a prescribed energy spectrum [41. In this configuration, turbulent scales and turbulence intensity change as the wall is approached. The integral length scale at the inflow plane is taken $7 \delta_{Z}$ 
which is about 0.1 of the domain length. A limited number of simulations have been conducted to assess the influence of the choice of the integral length scale at the inlet and this effect has been found to be negligible. The three-dimensional Navier-Stokes characteristic boundary condition (NSCBC) formulation 42 44 are imposed on the four lateral boundaries. To resolve the Kolmogorov scale and the flame structure everywhere, the cubic computational domain with dimension $L_{x_{i}}=L=70 \delta_{Z}$ is discretised by a uniform Cartesian mesh with total $256 \times 256 \times 256$ grid points, which ensures about 7 grid points per thermal flame thickness $\delta_{t h}=\frac{T_{b}-T_{u}}{\left.\max \left(\frac{\partial T}{\partial x}\right)\right|_{L}}$. Three cases are tested with different no-slip wall boundary conditions (case A: adiabatic wall boundary, case B: isothermal wall with $\tilde{T}_{w}=0.5$, case C: isothermal wall with $\tilde{T}_{w}=0.0$ ). Once the inflow with the mean velocity scans the entire domain $3 \sim 4$ times, the flow reaches the statistically stationary state. To ensure a good level of statistical convergence, totally 40 snapshots in a time span of $4 U_{1} / L$ are collected for the analysis presented in this paper.

\section{Results and analysis}

Fig. 2 shows instantaneous flow and scalar fields for three cases considered here. Overall the large scale flow structure is counterflow-like with the velocity component $u_{1}$ decreasing and tangential velocity components $u_{2}$ and $u_{3}$ increasing when the wall is approached along the wall-normal direction. For the present unity Lewis number case, the reaction progress variable and non-dimensional temperature for case A (adiabatic wall) assume the same spatial distribution. In contrast, cases B and C, with different wall heat losses, exhibit considerable differences between the non-dimensional temperature and the reaction progress fields. Especially, for case $\mathrm{C}$ with the lowest wall temperature $\tilde{T}_{w}=0.0$, the flame becomes broken because of the local flame quenching by excessive heat loss to the wall. Although for three cases with the same inflow feeding condition, cases B and C lose energy due to heat loss through the wall, which weakens the consumption rate in comparison to case $\mathrm{A}$, where combustion takes place under adiabatic conditions. Therefore in cases $\mathrm{B}$ and $\mathrm{C}$, the flame fronts tend to be closer to the wall. In the present analysis, $c=0.85$ isosurface is taken to be the flame surface because the maximum reaction rate takes place for this value of reaction progress variable $c$ for freely propagating flames. In the following sections, quantitative analyses of the FWI physics and the head-on flame quenching properties will be discussed.

\subsection{Local flame quenching and the wall heat flux}

Here the flame-wall distance is denoted as $\delta_{c=0.85}$, i.e. the distance from a local flame front to the wall along the wall-normal direction. The probablity density function (PDF) of $\delta_{c=0.85} / \delta_{z}$, the normalized flame-wall distance, is shown in Fig. 3. For case $\mathrm{A}$, on average the fame-wall distance is larger. From case B to C, becuase of more local quenching, flame fronts tend to be more spatially distrubted closer to the wall boundary. This quantitative PDF is consistent with the visualization in Fig. 2.

For caes $\mathrm{B}$ and $\mathrm{C}$ with wall heat loss, the local flame surface temperature changes in space. To address the local flame quenching, the mean flame temperature conditional on $\delta_{c=0.85} / \delta_{z}$ is plotted for three cases in Fig. 4. Here, the flame-wall distance is defined as the distance from the flame isosurface $c=0.85$ to the wall along the $x_{1}$ direction. In accordance with the spatial temperature distribution in Fig. 2 (a), the 


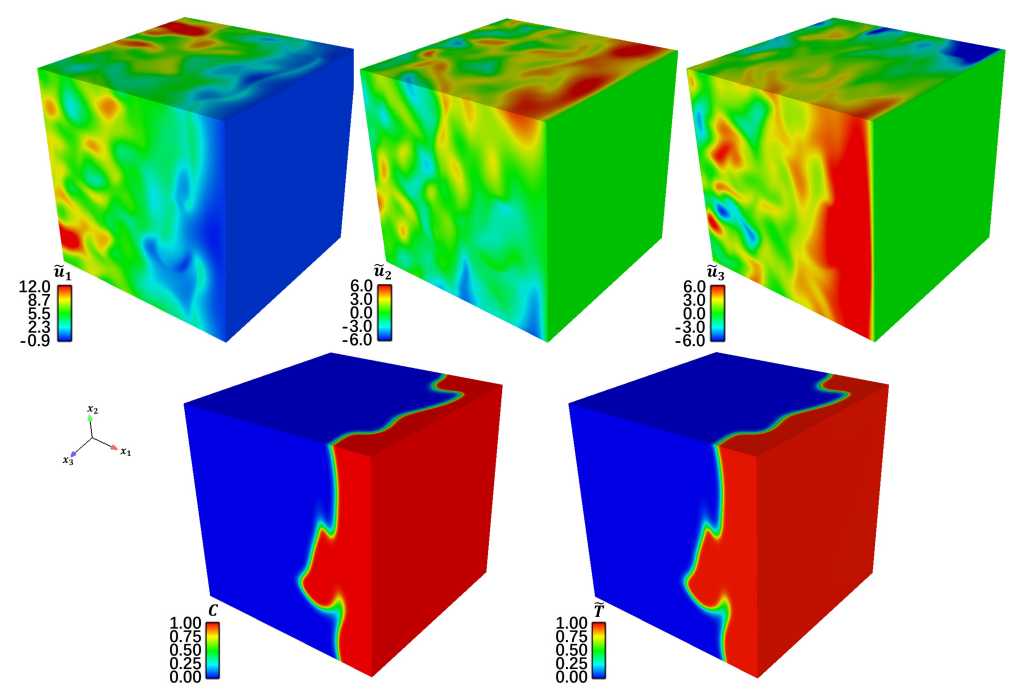

(a)

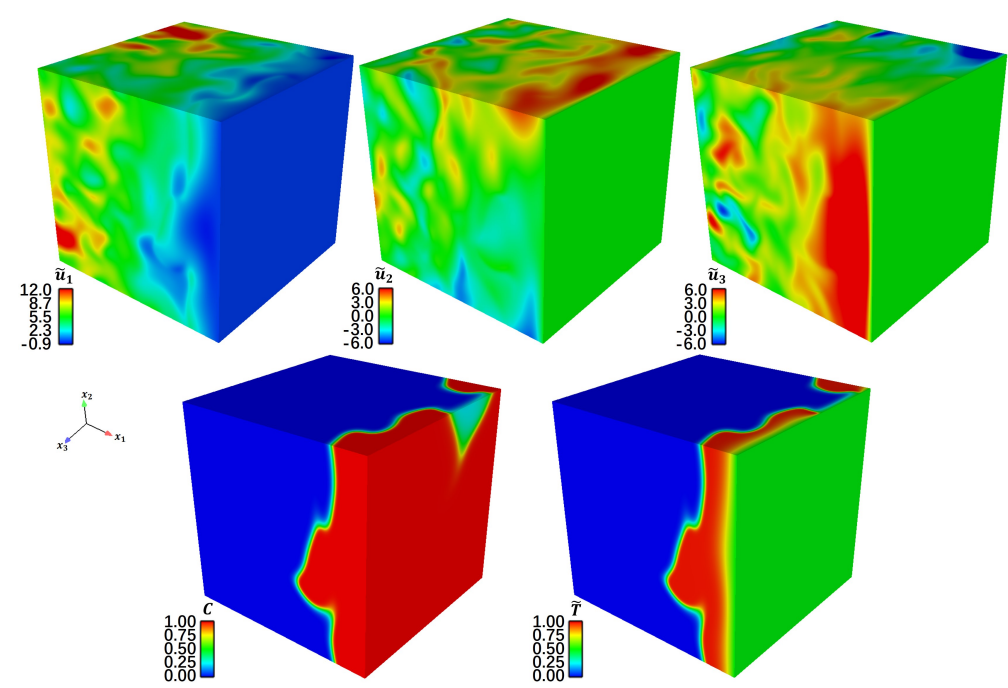

(b)

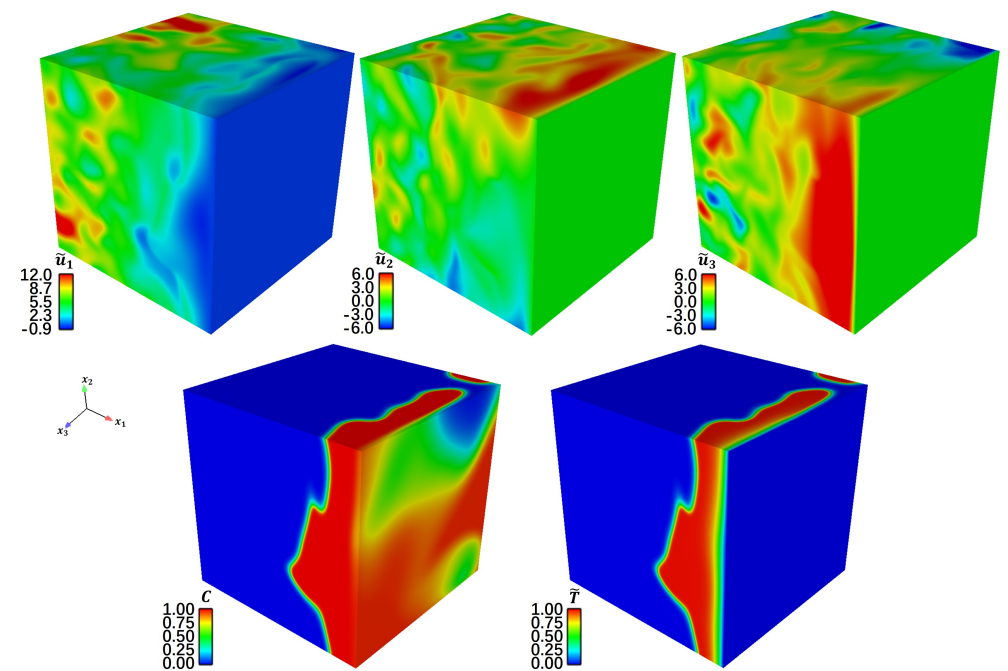

(c)

Figure 2. The flow and flame distribution for(a): case A with adiabatic wall, (b): case B with $\tilde{T}_{w}=0.5$ and (c): case $\mathrm{C}$ with $\tilde{T}_{w}=0.0$. 


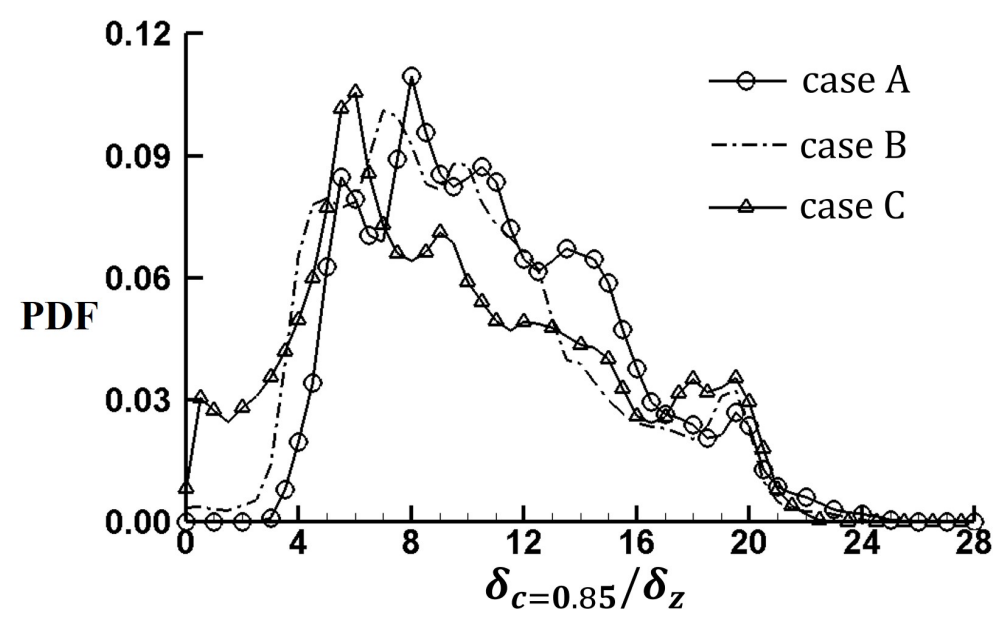

Figure 3. PDF of the normalized flame-wall distance $\delta_{c=0.85} / \delta_{z}$.

flame temperature in case $\mathrm{A}$ is equal to the $c$ value, i.e. 0.85. By contrast, case $\mathrm{B}$ and $\mathrm{C}$ exhibit a decrease in temperature from the adiabatic value away from the wall to the corresponding wall temperature, i.e. $\tilde{T}_{w}=0.5$ or $\tilde{T}_{w}=0.0$ at the wall. According to the temporally evolving FWI results in Poinsot et al.[12], the FWI zone can be divided into two sub-zones. For the present analysis, the similar sub-zones can be identified using the temperature distributions in Fig. 4 as:

(1)'quenching zone': the part from the cold wall to a distance with a critical Peclet number $P_{q}=\delta_{c=0.85} / \delta_{z}=2.5$. Fig. 4 suggests at this $P_{q}$, the temperature $\tilde{T}_{c=0.85}=0.6$, which is low enough to extinct the flame. Because of the very low or vanished heat release rate in this zone, as the flame-wall distance $\delta_{c=0.85} / \delta_{z}$ decreases, the temperature drops almost linearly from 0.6 to the temperature of the cold wall. The present critical Peclet number is comparable to the one proposed by Poinsot et al. [12] $\left(P_{q}=3.4\right)$ and Lai et al. $[13]\left(P_{q}=2.8\right)$.

(2) 'influence zone': the part in which the flame 'senses' the influences from the cold wall boundary. Fig. 4 indicates the influence zone can be quantified as $2.5<\delta_{c=0.85} / \delta_{z} \leq 8$. When $\delta_{c=0.85} / \delta_{z}>8$, the flame temperature is equal to the adiabatic value 0.85 but inside the influence zone the flame temperature starts to gently decrease.

Flame quenching can further be identified by the variation of chemical reaction rate when the flame approaches the wall. From the joint PDFs between the chemical reaction rate and the flame-wall distance shown in Fig. 5, we observe that for case A, the chemical reaction rate on the $c=0.85$ isosurface remains indepedent of $\delta_{c=0.85} / \delta_{z}$ because of the constant temperature on the isosurface, while for cases $\mathrm{B}$ and $\mathrm{C}$ the chemical reaction rate decreases rapidly in the influence zone because of the reduction of the flame temperature as a result of wall heat loss. When the wall temperature is not very low, as for case $\mathrm{B}$ with $\tilde{T}_{w}=0.5$, the chemical reaction rate assumes values larger than zero at the wall boundary. Differently, for case $\mathrm{C}$ the chemical reaction rate totally vanishes at $\delta_{c=0.85} / \delta_{z} \leq 2.5$.

Physically it is clear that the local flame quenching is the direct outcome of the heat loss to the wall boundary. To understand the statistical behaviour of this important quantity, Fig. 6 presents the joint PDFs between the normalized wall heat flux $\left.\tilde{Q}\right|_{w}$ and the flame-wall normal distance $\delta_{c=0.85} / \delta_{Z}$ for cases B and $\mathrm{C}$. As expected, the 


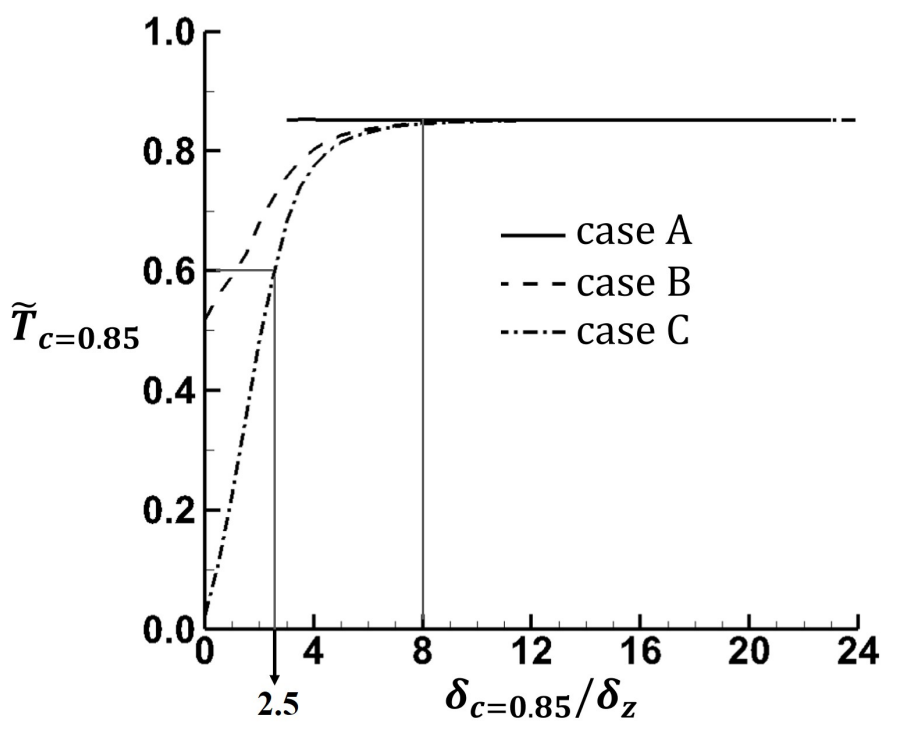

Figure 4. The variation of mean temperature $\tilde{T}_{c=0.85}$ on flame isosurface $c=0.85$ with the normalized flamewall distance $\delta_{c=0.85} / \delta_{z}$ for three cases.

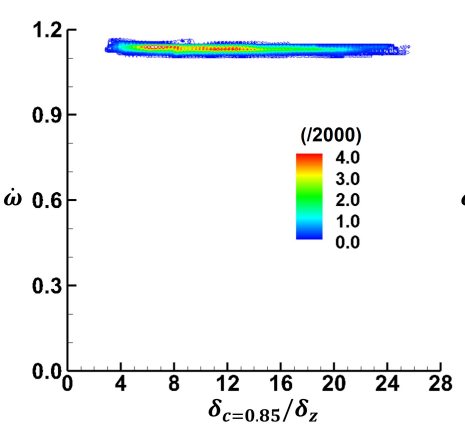

(a)

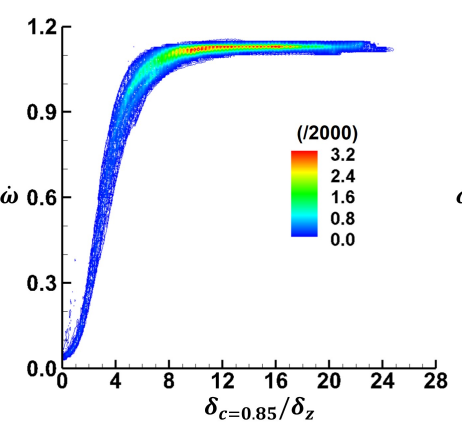

(b)

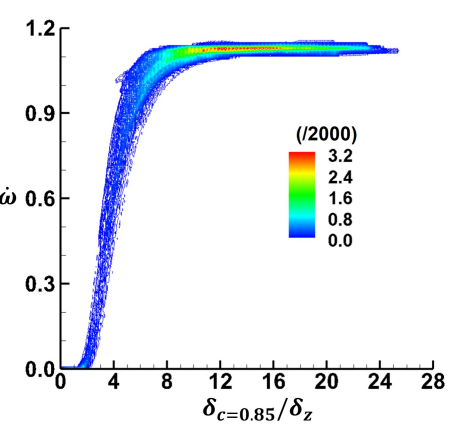

(c)

Figure 5. Joint PDFs between the chemical reaction rate and the flame-wall distance for for(a): case A with adiabatic wall, (b): case B with $\tilde{T}_{w}=0.5$ and (c): case $\mathrm{C}$ with $\tilde{T}_{w}=0.0$. 
heat flux increases with decreasing flame-wall distance until $\left.\tilde{Q}\right|_{w}$ attains a peak value, which occurs approximately at the critical Peclet number, e.g. $\delta_{c=0.85} / \delta_{z}=2.5$, for both cases B and C. Within the quenching zone, when $\delta_{c=0.85} / \delta_{z}$ continues to decrease, $\left.\tilde{Q}\right|_{w}$ decreases. This can be explained in the following manner. As $c=0.85$ isosurface approaches the cold wall, the heat loss through the wall reduces the rate of chemical reaction, giving rise to the decreases of the flame temperature and heat release rate. Quantitatively, the heat flux for case $\mathrm{C}$ ( with $\tilde{T}_{w}=0.0$ ) is in the range $0.2 \sim 0.4$, which is clearly greater than that for case B with $\left.\tilde{Q}\right|_{w}$ smaller than 0.2 . It is worth noting that the critical Peclet number at which the maximum heat flux magnitude is obtained is not significantly affected by the wall temperature but the wall boundary condition affects the magnitude of the maximum wall heat flux.

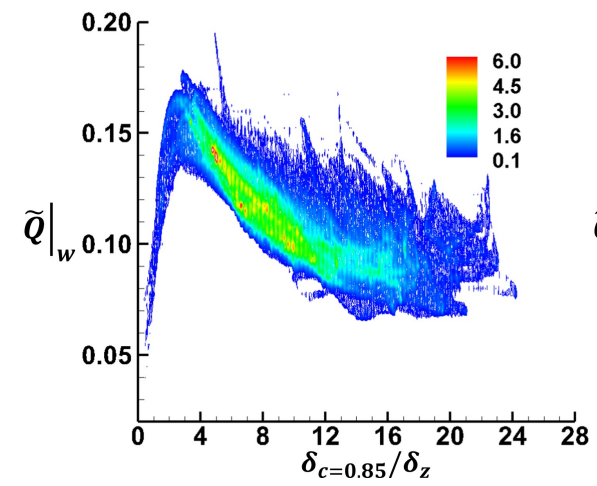

(a)

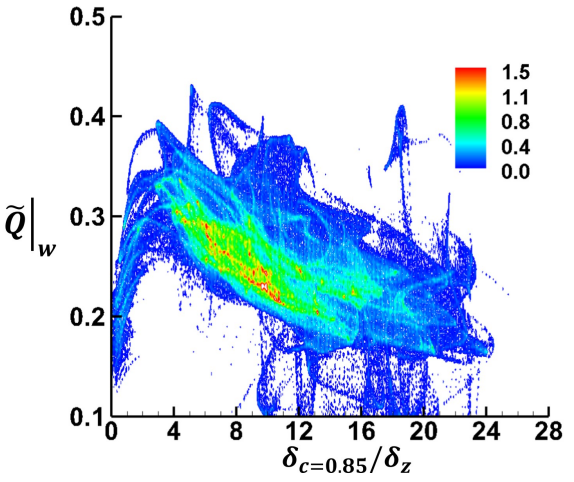

(b)

Figure 6. Joint PDFs between the heat loss to the wall and the flame wall distance for (a): case B with $\tilde{T}_{w}=0.5$ and $(\mathrm{b})$ : case $\mathrm{C}$ with $\tilde{T}_{w}=0.0$.

\section{2. $\quad$ Flame structure and flame dynamics}

To investigate the effect of FWI on the flame structure, Fig. 7 shows the distribution of the normalized vorticity magnitude $|\omega| \times \frac{\delta_{Z}}{S_{L}}$ at the $x_{2}=\frac{1}{2} L$ cross cut, where $|\omega|=\left(\omega_{i} \omega_{i}\right)^{1 / 2}$ with $\omega_{i}$ is the $i^{t h}$ component of vorticity. The $c=0.85$ isosurface is shown by the black lines. From the unburnt side to the burnt side, turbulence intensity decays rapidly because of the larger kinematic viscosity of the burnt gas and also due to supression of vorticity by dilatation within the flame. Moreover, in FWI, damping induced from the wall boundary is also important to decrease the turbulent intensity. Overall, both the thermal effect and the wall boundary can largely alter the flame structure. The similar phenomenon of the small-scale turbulence suppression by the flame has been reported by Hamlington et al.45] and Lai et al.[13. Poinsot et al. [12] demonstrated that the vortex pair plays a dominant role in the evolution of the flame structure.

To quantitatively examine the characteristics of the local flame structure, Fig. 8 shows the joint PDFs between the flame-wall distance $\delta_{c=0.85} / \delta_{z}$ and the normalized flame front curvature $K=\nabla \cdot \vec{n}$, where $\vec{n}=-\frac{\nabla c}{|\nabla c|}$ is the flame normal of the $c=0.85$ isosurface pointing to the unburned gas side. Overall, the flame curvature and the flame-wall distance are positively correlated. At the statistically stationary state, the downstream region between the flame front and wall is filled with the burnt product, 


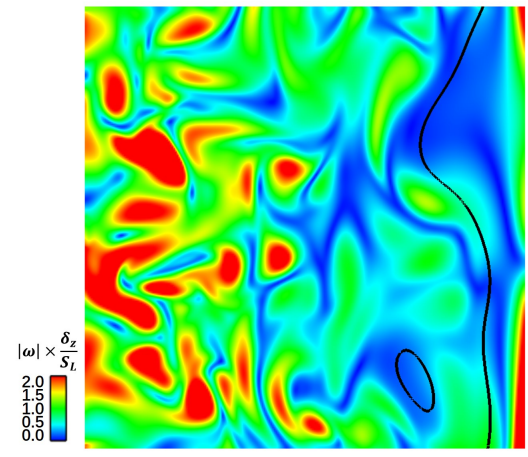

(a)

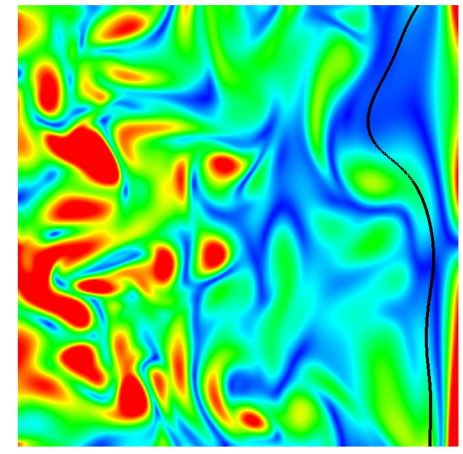

(b)

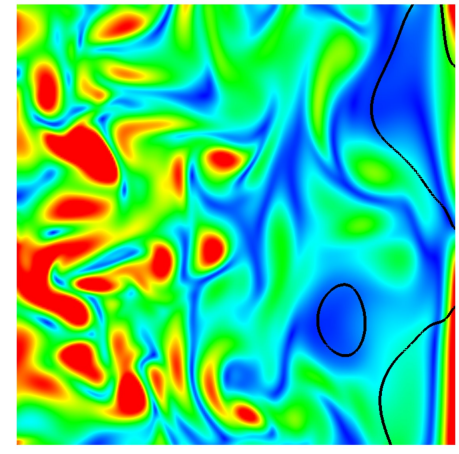

(c)

Figure 7. Distribution of normalised vorticity magnitude $|\omega| \times \frac{\delta_{Z}}{S_{L}}$ in the cross-cut $x_{2}=\frac{1}{2} L$ plane for(a): case A with adiabatic wall, (b): case B with $\tilde{T}_{w}=0.5$ and $(\mathrm{c})$ : case $\mathrm{C}$ with $\tilde{T}_{w}=0.0$. Thick black lines denote the flame fronts.

while the fresh gas is mostly on the upstream side. Geometrically the isosurfaces with positive curvature are inclined to bend toward the fresh gas side, and thus are probably far from the wall. In contrast the isosurfaces with negative curvature tend to bend toward the burnt gas side with smaller flame-wall distance. Clearly, as the flame-wall distance $\delta_{c=0.85} / \delta_{z}$ decreases, the flame curvature for the three cases shifts from being positively skewed to being negatively skewed (see Fig. 8). An important observation is that an additional tail of the joint PDF in Fig. 8(b) and (c) can be seen in cases B and $\mathrm{C}$ in the quenching zone. Such behavior can be explained in the following manner. At very small flame-wall distance, e.g. $\delta_{c=0.85} / \delta_{z}<1$ in Fig. 8 (b) and (c), the flame front locally breaks, as marked by the two boxes in Fig. 9 . The zoomed in flame front topology indicates that under such a condition the $c$ value locally can be large because of flame extinction. Consequently the flame front bends to have positive curvature values. Such tail exists for both case B and $\mathrm{C}$ with broken flame fronts in the nearwall region. The tail of the joint $\mathrm{PDF}$ between $\delta_{c=0.85} / \delta_{z}$ and $K=\nabla \cdot \vec{n}$ in case $\mathrm{B}$ is much smaller than in case $\mathrm{C}$ because of much weaker local flame quenching.

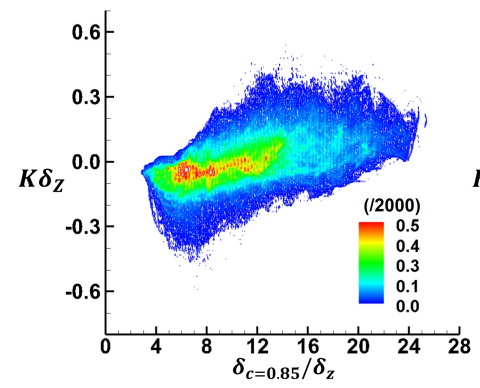

(a)

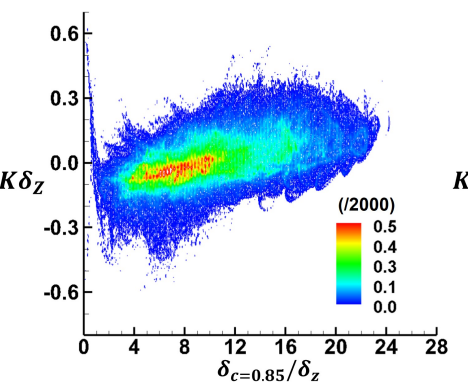

(b)

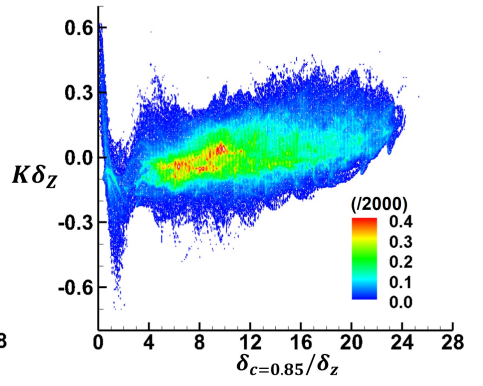

(c)

Figure 8. Joint PDFs between the curvature and the flame-wall distance for(a): case A with adiabatic wall, (b): case B with $\tilde{T}_{w}=0.5$ and (c): case $\mathrm{C}$ with $\tilde{T}_{w}=0.0$.

The dilatation $\Delta=\partial u_{i} / \partial x_{i}$, is another important quantity, which is directly affected by FWI. Fig. 10 shows that curvature and dilatation are negatively correlated, and the nature of correlation is markedly different for positive and negative curvatures. Considering the flame isosurface $c=0.85$, for case A with the adiabatic wall, the joint $\mathrm{PDF}$ is relative simple, showing a negative correlation between $\Delta$ and $K$. For cases $\mathrm{B}$ and $\mathrm{C}$, because of local flame quenching, another branch appears for negative 


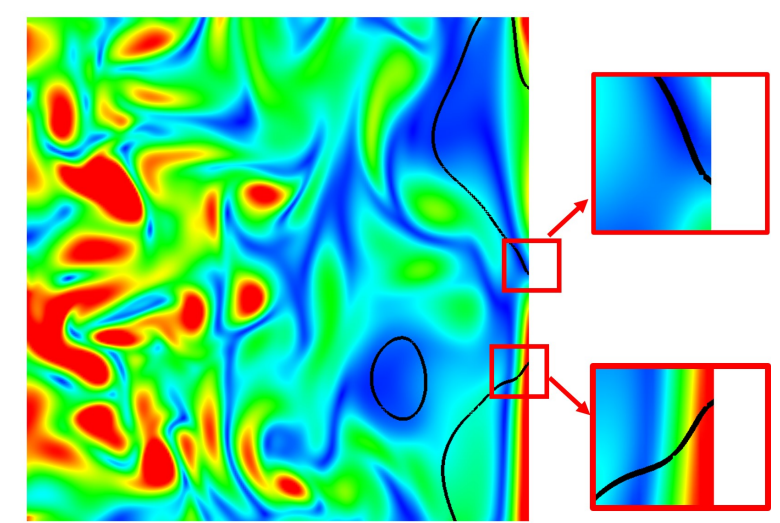

Figure 9. The magnified view of the broken flame topology of flame isosurface $c=0.85$ in the quenching zone for case $\mathrm{C}$ with $\tilde{T}_{w}=0.0$.

dilatation samples.

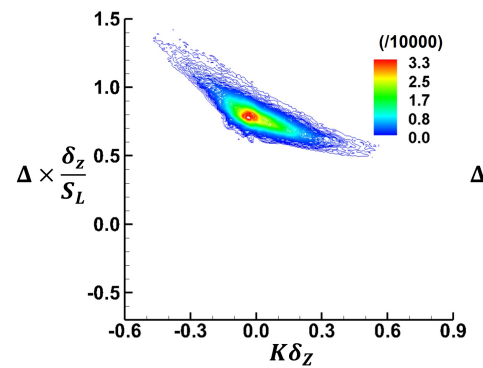

(a)

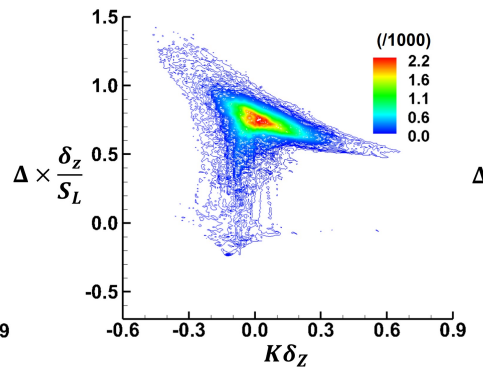

(b)

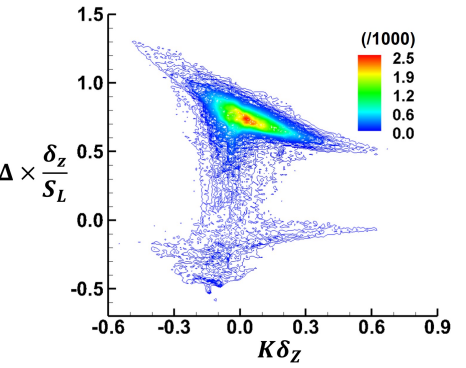

(c)

Figure 10. Joint PDFs between the curvature and the dilatation on the flame isosurface $c=0.85$ for(a): case A with adiabatic wall, (b): case B with $\tilde{T}_{w}=0.5$ and (c): case $\mathrm{C}$ with $\tilde{T}_{w}=0.0$.

It is worth noting that $\Delta=s_{t}+s_{n}$, where $s_{t}=\left(\delta_{i j}-n_{i} n_{i}\right) \frac{\partial u_{i}}{\partial x_{j}}$ and $s_{n}=n_{i} n_{i} \frac{\partial u_{i}}{\partial x_{j}}$ are flame-tangential strain rate and flame-normal strain rate, respectively. Thus, the statistical behaviours of dilatation and its correlation with curvature can further be investigated by analysing the $s_{t}$ and $s_{n}$ statistics separately. Fig. 11] shows joint PDFs of the curvature and the flame-tangential strain rate on the flame isosurface $c=0.85$ for three cases. Some previous studies [46,47] reported no correlation between tangential strain rate and curvature, whereas negative correlation was reported in other analyses [48 52]. Chakraborty et al. [50 52] showed the correlation strength weakens with decreasing (increasing) $\tau\left(u^{\prime} / S_{L}\right)$. In FWI, when the normalized flame tangential strain rate is small, i.e. $s_{t} \delta_{z} / S_{L}<0.3$, the flame front curvature and the flame-tangential strain rate are negatively correlated; while when $s_{t} \delta_{z} / S_{L} \geq 0.3$ such correlation is much weaker or even vanishingly small. For the positive curvature samples, the flame elements have relatively large flame-wall distance as they bend toward the unburned gas side. Under the counter-flow like configuration, the strain rate is roughly inversely proportional to the distance from the wall. Therefore the larger positive curvature samples correspond to small flame-tangential strain rate. Interestingly, for all the three cases, such correlation relation remains almost the same, even quantitatively, i.e. independent of the wall boundary condition and local flame quenching, which indicates that the such correlation is primarily determined by the interaction between the flame and turbulence. 


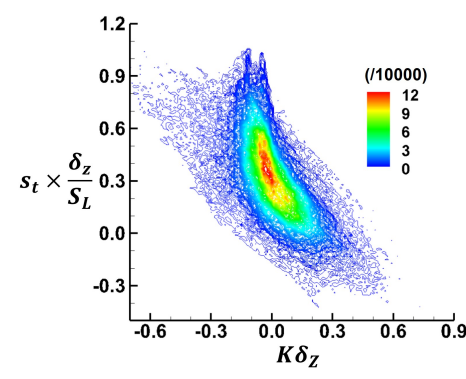

(a)

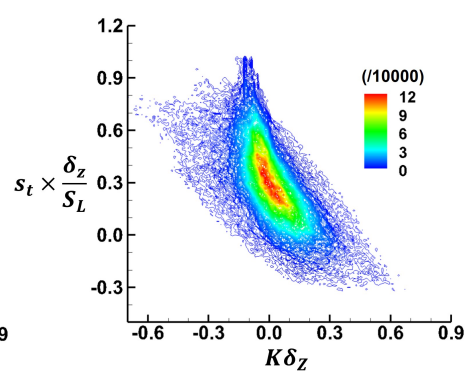

(b)

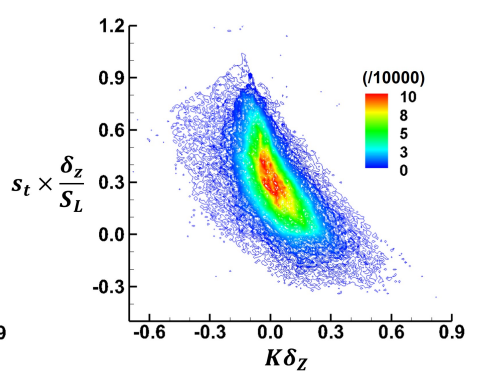

(c)

Figure 11. Joint PDFs between the curvature and the flame-tangential strain rate on the flame isosurface $c=0.85$ for(a): case A with adiabatic wall, (b): case B with $\tilde{T}_{w}=0.5$ and (c): case C with $\tilde{T}_{w}=0.0$.

In contrast, as shown in Fig. 12, the joint PDFs of curvature and the flame-normal strain rate for these three cases are largely different. For the adiabatic wall case, the normal strain rate remains mostly positive because of the local thermal expansion. For all three cases, the part with positive $s_{n}$ remains almost unchanged. However, for cases B and C, larger the wall heat loss is, bigger the negative normal strain rate part becomes. Therefore the flame fronts with negative normal strain rate are associated with low flame temperatures and are expected to be close to the wall, where the flame surface curvature is mostly negative, as shown in the joint PDFs. From $\Delta=s_{t}+s_{n}$, it suggests that the extra branch of the dilatation-curvature joint PDF for negative dilatation values is mainly from the contribution of negative $s_{n}$. Here, $s_{n}$ and the curvature are found to be slightly positively correlated.

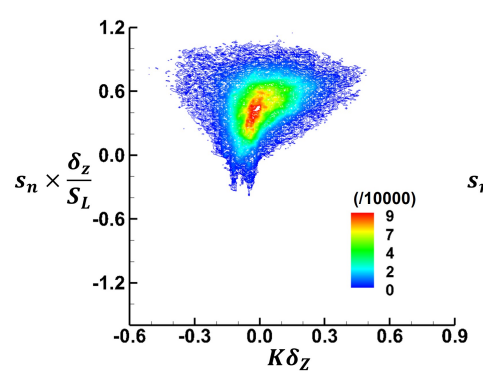

(a)

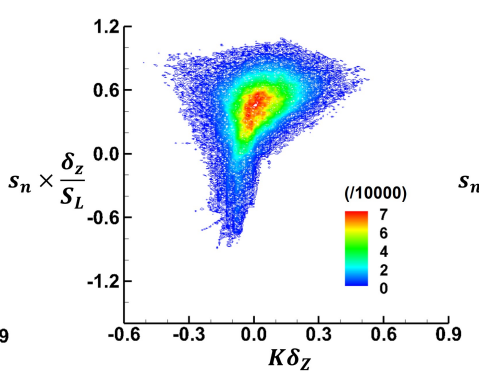

(b)

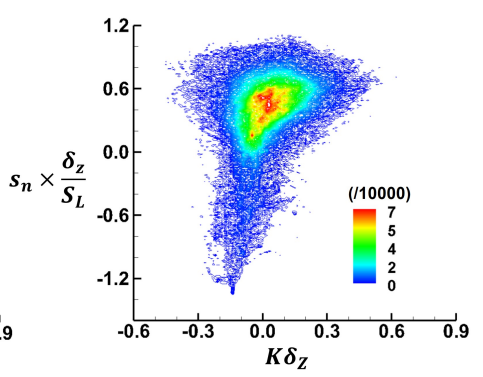

(c)

Figure 12. Joint PDFs between the curvature and the flame-normal strain rate on the flame isosurface $c=0.85$ for(a): case A with adiabatic wall, (b): case B with $\tilde{T}_{w}=0.5$ and (c): case $\mathrm{C}$ with $\tilde{T}_{w}=0.0$.

\subsection{Scalar gradient orientation statistics}

Here, the principle strain rates are defined as $\Lambda_{i}(i=1,2,3)$, and $\vec{\lambda}_{i}(i=1,2,3)$ are the corresponding eigenvectors, i.e. the principal axes. Let $\Lambda_{1}>\Lambda_{2}>\Lambda_{3}$, which represent the most extensive, the intermediate and the most compressive principle strain rates, respectively. The statistical behaviour of $s_{n}=\Lambda_{i} \cos ^{2}<\vec{n}, \overrightarrow{\lambda_{i}}>$ depends on the flame normal vector $\vec{n}$ and $\vec{\lambda}_{i}(i=1,2,3)$. For the passive scalar mixing, the scalar gradient predominantly aligns with and the most compressive principal axis 53 56]. However, in reactive turbulence the heat release changes such orientation substantially (i.e. a preferential alignment between $|\nabla c|$ and $\lambda_{1}$ is observed when heat release effects overcome turbulent straining) 4555760. For FWI, it can reasonably be expected that the wall influence may introduce more complexity. 
To understand the orientation relations across the the flame front, different $c$ isosurfaces have been investigated, including the $c=0.05$ in the fresh gas side and $c=0.95$ in the product gas side, together with the $c=0.6$ isosurface for the sake of the maximum dilatation. Fig. 13 shows conditional on $c=0.6$ (in the reaction zone) the PDFs of $\left|\cos \left\langle\vec{n}, \vec{\lambda}_{i}\right\rangle\right|$ for the three cases. Apparently, because of the dominant role of thermal expansion in the flame zone, the flame normal $\vec{n}$ is mostly oriented along the most extensive principal axis, and misaligns with the intermediate and most compressive principle axes, i.e. $\vec{\lambda}_{2}$ and $\vec{\lambda}_{3}$. Meanwhile it should be noted in Fig. 13 that $\vec{n}$ and $\vec{\lambda}_{3}$ tend to align to some extent in cases B and C, which indicates the effects of heat release weaken due to heat loss through the wall and this gives rise to a (an) reduction (increase) in the extent of collinear alignment between $\overrightarrow{\lambda_{1}}\left(\overrightarrow{\lambda_{3}}\right)$.
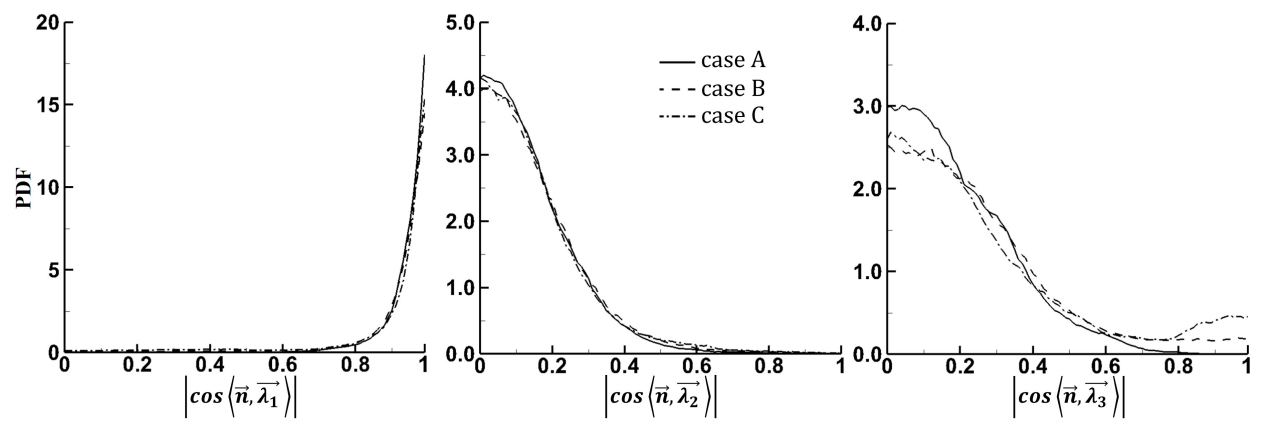

Figure 13. PDFs of the alignment between the flame normal and principle axes $\left(\left|\cos \left(\vec{n}, \vec{\lambda}_{1}\right)\right|,\left|\cos \left(\vec{n}, \vec{\lambda}_{2}\right)\right|\right.$, $\left.\left|\cos \left(\vec{n}, \vec{\lambda}_{3}\right)\right|\right)$ on the flame isosurface $c=0.6$ for the three cases.

Fig. 14 shows joint PDFs between $\Delta$ and $s_{n}$ for $c=0.6$ isosurface for all three cases considered here. The thermal expansion generates large values of positive dilatation. Therefore in the reaction zone, $s_{n}$ and the flame dilatation are strongly positively correlated, which is consistent with the alignment between the flame normal and the most extensive principle axis in Fig. 13. For cases B and C, because of the wall heat loss, samples with the negative flame-normal strain rate appear, which give rise to tails of the joint PDFs in Fig. 14 (b) and (c). Especially for case C, as has been discussed in the context of Fig. 10(c) and Fig. 12(c), negative dilatation and negative flame-normal strain rate samples are generated by local flame quenching, which is consistent with the small probability of $\left|\cos \left\langle\vec{n}, \vec{\lambda}_{3}\right\rangle\right|=1$ in Fig. 13 (c). No clear correlation between $s_{n}$ and the flame dilatation has been observed in the tail of the joint PDF.

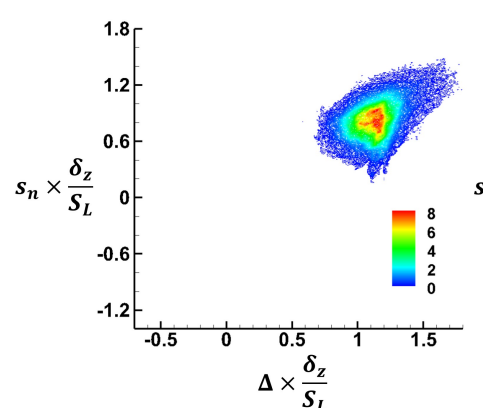

(a)

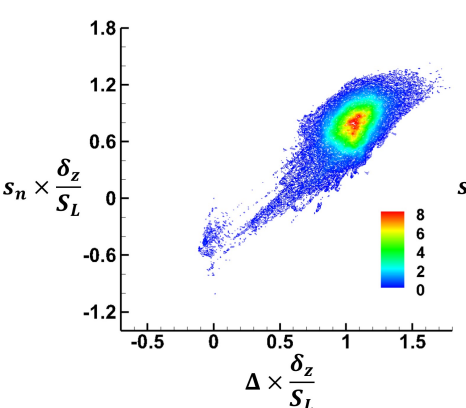

(b)

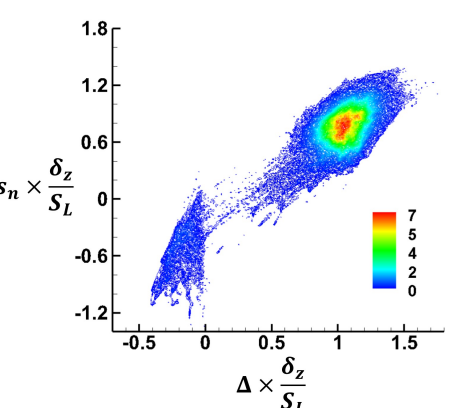

(c)

Figure 14. Joint PDFs between the dilatation and the flame-normal strain rate on the flame isosurface $c=0.6$ for (a): case A, (b): case B and (c): case C. 
The same joint PDF has also been investigated conditional on the fresh gas side $c=0.05$ isosurface and the product side $c=0.95$ isosurface. As shown in both Fig. 15 and Fig. 16, for different cases the main parts of these joint PDFs are similar. In the unburned gas side, $s_{n}$ and $\Delta$ are weakly negative correlated, while no clear correlation can be observed in Fig. 16. Because of the weak chemical reaction rate, $s_{n}$ and $\Delta$ assume much smaller magnitudes than these quantities in the reaction zone. Moreover, from case A to case $\mathrm{C}$, stronger flame quenching brings more negative dilatation samples.

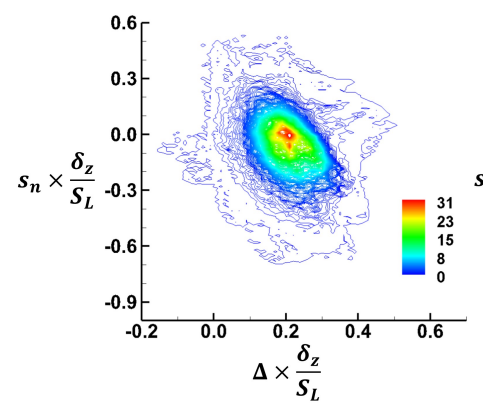

(a)

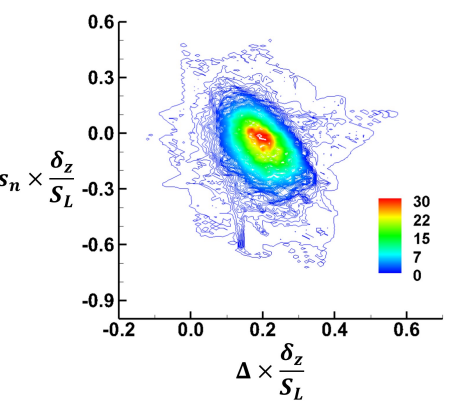

(b)

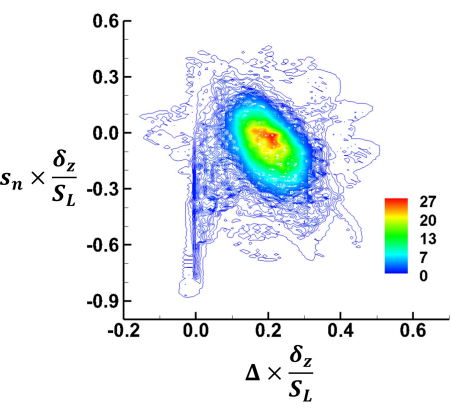

(c)

Figure 15. Joint PDFs between the dilatation and the flame-normal strain rate on the flame isosurface $c=0.05$ for (a): case A, (b): case B and (c): case C.

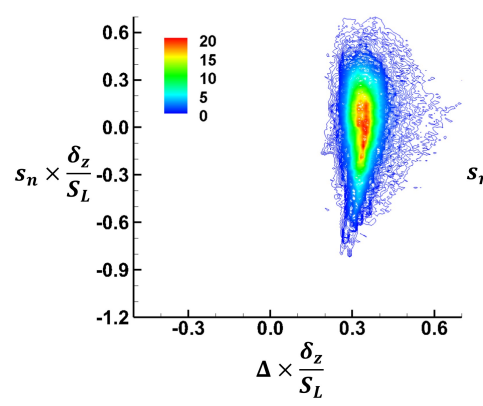

(a)

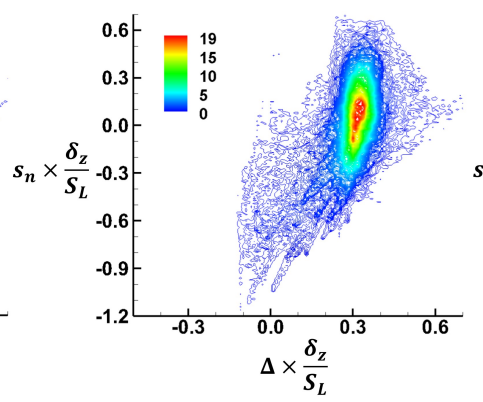

(b)

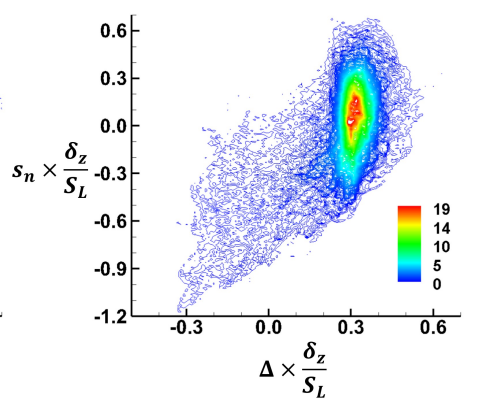

(c)

Figure 16. Joint PDFs between the dilatation and the flame-normal strain rate on the flame isosurface $c=0.95$ for (a): case A, (b): case B and (c): case C.

In the same vein of Fig. 13, the alignment statistics conditional on $c=0.05$ in the fresh gas side are presented in Fig. 17. For the sake of brevity, the similar results conditional on $c=0.95$ are omitted. Very differently from the reaction zone results, when the heat release rate is small, the $c$ field behaves similarly to a passive scalar, whose isosurface normal is then preferentially aligned with the most compressive principal axis $\vec{\lambda}_{3}$, rather than the most extensive principal axis $\vec{\lambda}_{1}$. On the $c=0.95$ isosurface the orientation between $\vec{n}$ and $\vec{\lambda}_{1}$ looks even more random. In different flame zones, the misalignment relation between $\vec{n}$ and the intermediate principal axis $\vec{\lambda}_{2}$ remains qualitatively similar.

\section{Conclusions}

The flame-wall interaction (FWI) of statistically planar premixed turbulent flames has been analysed using direct numerical simulation (DNS) in a head-on flame quenching 

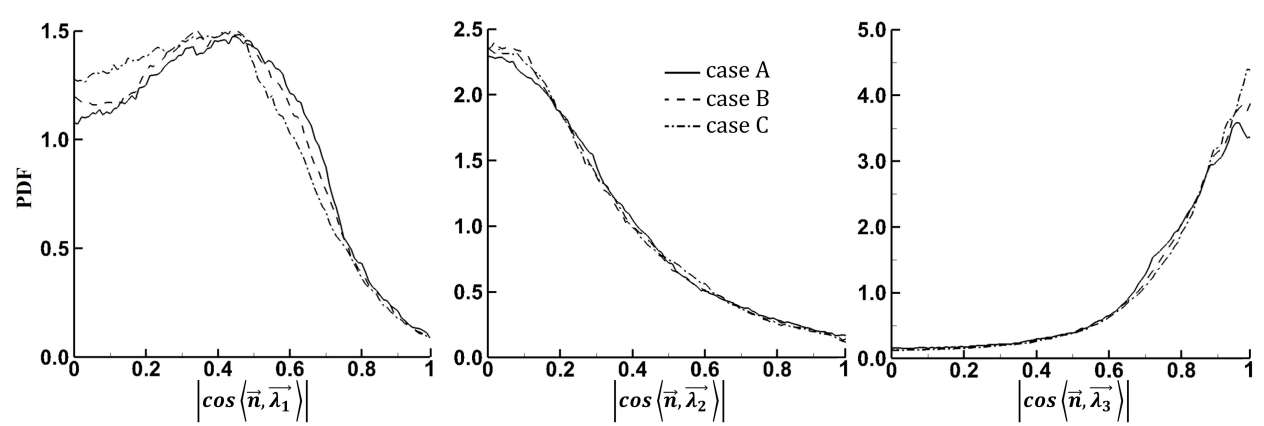

Figure 17. PDFs of the alignment between the flame normal and principle axes $\left(\left|\cos \left(\vec{n}, \vec{\lambda}_{1}\right)\right|,\left|\cos \left(\vec{n}, \vec{\lambda}_{2}\right)\right|\right.$, $\left.\left|\cos \left(\vec{n}, \vec{\lambda}_{3}\right)\right|\right)$ on the flame isosurface $c=0.05$ for the three cases.

configuration which is at the statistically stationary state. The analysis of FWI has been carried out for different wall boundary conditions and its influences on the flame structure have been analyzed in detail. It has been found that the flame approaches closer to the wall and becomes more spatially distributed for lower wall temperatures. Moreover, the flame front becomes broken as a result of local flame quenching due to heat loss through the wall. The FWI zone is split into two sub-zones, i.e. influence and quenching zones, which are the regions in which the flame senses the presence of the wall and ceases to exist due to quenching, respectively. The normalised flame quenching distance for the statistically stationary configuration has been found to be consistent with previous findings in the unsteady HOQ configurations. Moreover, it has been found that the normalised wall heat flux is affected by the wall boundary condition but the normalised wall normal distance where the wall heat flux assumes its peak magnitude remains unaffected by the wall temperature.

Correlations between important flame parameters, such as the flame curvature, flame-wall distance, flame dilatation, including the flame tangential strain rate and the flame normal strain rate have been investigated in detail. In this configuration the flame curvature and the flame-wall distance have been found to be positively correlated. As the flame-wall distance decreases, the flame curvature shifts from being positively skewed to being negatively skewed in all cases considered here, which has been explained in terms of the spatial distribution of the reactant and products and heat loss through the wall. It has been found that FWI has significant influences on the statistical distributions of tangential and flame normal strain rates. The strength of dilatation rate weakens as the wall is approached, which also affects the tangential strain rate and acts to reduce the extent of collinear alignment of reactive scalar gradient with the eigenvector corresponding to the most extensive principal strain rate. This alignment behaviour in turn affects the near-wall behaviour of the flame normal strain rate.

Although the statistics of wall heat flux, quenching distance, and different strain rates are principally driven by heat transfer and fluid-dynamics and not by chemical mechanism, further analysis will be necessary in the presence of detailed chemistry and transport for higher values of turbulent Reynolds number than the values analysed here for deeper physical understanding. 


\section{Acknowledgements}

LW thanks for the financial support of NSFC (under the grant No. 91441116), and partially by the Sino-French (NSFC-CNRS) joint research project (No. 11611130099, NSFC China, and PRC 2016-2018 LATUMAR "Turbulence lagrangienne: études numériques et applications environnementales marines", CNRS, France). The computational support from the Center for High Performance Computing at Shanghai Jiao Tong University is gratefully appreciated. NC acknowledges the financial and computational supports of EPSRC (EP/K025163/1) and ARCHER,respectively.

\section{Appendix. Effects of temperature-dependent thermo-physical properties}

To compare the simulation results using temperature dependent thermo-properties , the Sutherlands law is adopted for the dynamics viscosity as $\mu=$ $\mu_{r e f}\left(T / T_{r e f}\right)^{3 / 2}\left(T_{r e f}+S\right) /(T+S)$, where $S$ is the Sutherland temperature (correspondingly the thermal conductivity and mass diffusivity are temperature dependent as well). Fig. 1 shows comparison of the flame-wall distance dependence of the chemical reaction rate, from the temperature dependent and constant thermo-physical properties in case C. As it can be seen, the difference does exist, but only in a quantitative sense, which means the main physics remains valid, at least in a qualitative sense.

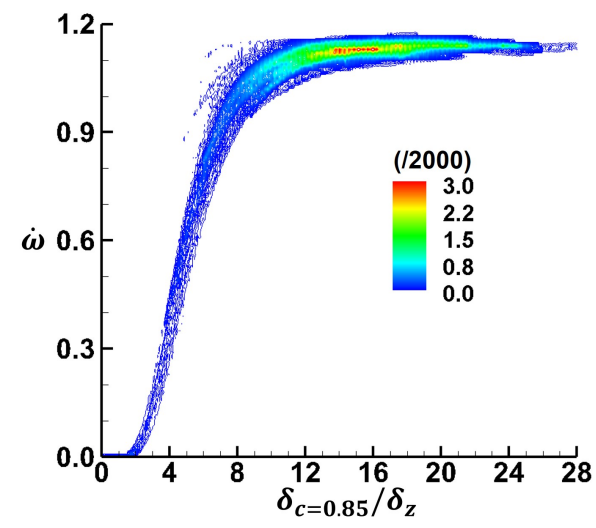

(a)

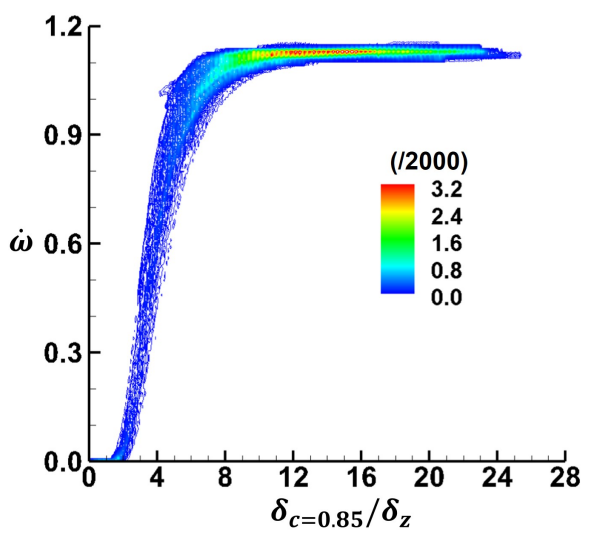

(b)

Figure 1. Joint PDFs between the flame-wall distance and the reaction rate for case $\mathrm{C}$ using (a) temperature dependent thermo-physical properties, (b) constant thermo-physical properties.

\section{References}

[1] R.S. Cant, S.B. Pope, and K.N. Bray, Modelling of flamelet surface-to-volume ratio in turbulent premixed combustion, Symp. (Intl) on Combustion 23 (1990), pp. 809-815.

[2] N. Peters, The turbulent burning velocity for large-scale and small-scale turbulence, J. Fluid Mech. 384 (1999), pp. 107-132.

[3] D. Veynante and L. Vervisch, Turbulent combustion modeling, Prog. Energy Combust. Sci. 28 (2002), pp. 193-266.

[4] H. Pitsch, Large eddy simulation of turbulent combustion, Annu. Rev. Fluid Mech. 38 (2006), pp. 453-482. 
[5] N. Chakraborty and M. Klein, A priori direct numerical simulation assessment of algebraic flame surface density models for turbulent premixed flames in the context of large eddy simulation, Phys. Fluids 20 (2008), p. 085108.

[6] N. Chakraborty and R.S. Cant, Direct numerical simulation analysis of the Flame Surface Density transport equation in the context of Large Eddy Simulation, Proc. Combust. Inst. 32 (2009), pp. 1445-1453.

[7] A.A. Putnam and R.A. Jensen, Application of dimensionless numbers to flash-back and other combustion phenomena, Symp. on Combustion and Flame, and Explosion Phenomena 3 (1948), pp. 89-98.

[8] J. Jarosiński, Flame quenching by a cold wall, Combust. Flame 50 (1983), pp. 167-175.

[9] P.W. Fairchild, R.D. Fleeter, and F.E. Fendell, Raman spectroscopy measurements of flame quenching in a duct-type crevice, Symp. (Intl) on Combustion 20 (1985), pp. 85-90.

[10] J. Jarosiński, A survey of recent studies on flame extinction, Prog. Energy Combust. Sci. 12 (1986), pp. 81-116.

[11] B. Lewis and G.V. Elbe, Combustion waves in laminar flow - combustion, flames and explosions of gases (third edition) - chatpter $V$, Combustion Flames and Explosions of Gases (1987), pp. 215-417.

[12] T. Poinsot, D.C. Haworth, and G. Bruneaux, Direct simulation and modeling of flame-wall interaction for premixed turbulent combustion, Combust. Flame 95 (1993), pp. 118-132.

[13] J. Lai, N. Chakraborty, and A. Lipatnikov, Statistical behaviour of vorticity and enstrophy transport in head-on quenching of turbulent premixed flames, European Journal of Mechanics - B/Fluids (2016).

[14] F. Dabireau, B. Cuenot, O. Vermorel, and T. Poinsot, Interaction of flames of H2 + O2 with inert walls, Combust. Flame 135 (2003), pp. 123-133.

[15] J. Lai and N. Chakraborty, Effects of Lewis number on head on quenching of turbulent premixed flames: A Direct Numerical Simulation analysis, Flow, Turbul. Combust. 96 (2016), pp. 279-308.

[16] C.K. Westbrook, A.A. Adamczyk, and G.A. Lavoie, A numerical study of laminar flame wall quenching, Combust. Flame 40 (1981), pp. 81-99.

[17] O. Ezekoye, R. Greif, and R.F. Sawyer, Increased surface temperature effects on wall heat transfer during unsteady flame quenching, Symp. (Intl) on Combustion 24 (1992), pp. 1465-1472.

[18] P. Popp and M. Baum, Analysis of wall heat fluxes, reaction mechanisms, and unburnt hydrocarbons during the head-on quenching of a laminar methane flame, Combust. Flame 108 (1997), pp. 327-348.

[19] R. Friedman and W.C. Johnston, The wall-quenching of laminar propane flames as a function of pressure, temperature, and air-fuel ratio, J. Appl. Phys. 21 (1950), pp. 791795.

[20] S. Labuda, M. Karrer, J. Sotton, and M. Bellenoue, Experimental study of single-wall flame quenching at high pressures, Combust. Sci. Technol. 183 (2011), pp. 409-426.

[21] W.A. Daniel, Flame quenching at the walls of an internal combustion engine, Symp. (Intl) on Combustion 6 (1957), pp. 886-894.

[22] A. Gruber, R. Sankaran, E.R. Hawkes, and J.H. Chen, Turbulent flame-wall interaction: a direct numerical simulation study, J. Fluid Mech. 658 (2010), pp. 5-32.

[23] M. Mann, C. Jainski, M. Euler, B. Bhm, and A. Dreizler, Transient flamewall interactions: Experimental analysis using spectroscopic temperature and co concentration measurements, Combust. Flame 161 (2014), pp. 2371-2386.

[24] K. Jenkins and R.S. Cant, Direct numerical simulation of turbulent flame kernels, Recent Advances in DNS and LES. Springer Netherlands (1999), pp. 191-202.

[25] A.A. Wray, Minimal storage time-advancement schemes for spectral methods, in NASA Ames Research Center, Moffett Field. 1991.

[26] T. Poinsot and D. Veynante, Theoretical and Numerical Combustion, R.t.edwards Inc, 2005.

[27] O. Ezekoye and R. Greif, A comparison of one and two dimensional flame quenching: 
Heat transfer results, in National conference and exposition on heat transfer, Atlanta, GA (United States). 1993.

[28] G. Bruneaux, K. Akselvoll, T. Poinsot, and J.H. Ferziger, Flame-wall interaction simulation in a turbulent channel flow, Combust. Flame 107 (1996), pp. 27-44.

[29] G. Bruneaux, T. Poinsot, and J.H. Ferziger, Premixed flame-wall interaction in a turbulent channel flow: budget for the flame surface density evolution equation and modelling, J. Fluid Mech. 349 (1997), pp. 191-219.

[30] C. Jainski, M. Rismann, B. Bohm, and A. Dreizler, Experimental investigation of flame surface density and mean reaction rate during flamewall interaction, Proc. Combust. Inst. 36 (2017), pp. 1827-1834.

[31] J. Lai, M. Klein, and N. Chakraborty, Direct numerical simulation of head-on quenching of statistically turbulent premixed methane-air flames using a detailed chemical mechanism, Proceedings of 10th Mediterranean Combustion Symposium,17th -21st September, 2017 (2017).

[32] T. Poinsot, T. Echekki, and M.G. Mungal, A study of the laminar flame tip and implications for premixed turbulent combustion, Combust. Sci. Technol. 81 (1992), pp. 45-73.

[33] D.S. Louch and K.N.C. Bray, Vorticity in unsteady premixed flames: vortex pairpremixed flame interactions under imposed body forces and various degrees of heat release and laminar flame thickness, Combust. Flame 125 (2001), pp. 1279-1309.

[34] A.J. Aspden, A numerical study of diffusive effects in turbulent lean premixed hydrogen flames, Proc. Combust. Inst. 36 (2017), pp. 1997-2004.

[35] S.R. Vosen, R. Greif, and C.K. Westbrook, Unsteady heat transfer during laminar flame quenching, Symp. (Intl) on Combustion 20 (1985), pp. 75-83.

[36] L. Connelly, T. Ogasawara, D. Lee, R. Greif, and R.F. Sawyer, Fall meeting, The Combustion Institute/Western States Section, Stanford, CA, Paper N. WSCI 93-077 (1993).

[37] T.M. Alshaalan and C.J. Rutland, Turbulence, scalar transport, and reaction rates in flame-wall interaction, Proc. Combust. Inst. 27 (1998), pp. 793-799.

[38] T. Alshaalan and C.J. Rutland, Wall heat flux in turbulent premixed reacting flow, Combust. Sci. Technol. 174 (2002), pp. 135-165.

[39] R.S. Cant, C.J. Rutland, and A. Trouvé, Statistics for laminar flamelet modeling, Proceedings of the Summer Program 1990, Center for Turbulence Research, Stanford, pp. 271-279 (1990)

[40] T.D. Dunstan, N. Swaminathan, and K.N.C. Bray, Influence of flame geometry on turbulent premixed flame propagation: a DNS investigation, J. Fluid Mech. 709 (2012), pp. 191-222.

[41] R.S. Rogallo, Numerical experiments in homogeneous turbulence, Nasa Sti/recon Technical Report N 81 (1981).

[42] C.S. Yoo, Y. Wang, A. Trouvé, and H.G. Im, Characteristic boundary conditions for direct simulations of turbulent counterflow flames, Combust. Theory Modell. 9 (2005), pp. $617-646$.

[43] C.S. Yoo and H.G. Im, Characteristic boundary conditions for simulations of compressible reacting flows with multi-dimensional, viscous and reaction effects, Combust. Theory Modell. 11 (2007), pp. 259-286.

[44] G. Lodato, P. Domingo, and L. Vervisch, Three-dimensional boundary conditions for direct and large-eddy simulation of compressible viscous flows, J. Comput. Phys. 227 (2008), pp. 5105-5143.

[45] P.E. Hamlington, A.Y. Poludnenko, and E.S. Oran, Interactions between turbulence and flames in premixed reacting flows, Phys. Fluids 23 (2011), p. 125111.

[46] R. Cant, C. Rutland, and A. Trouvé, Statistics for laminar flamelet modelling, Proc. of the Summer Program 1990, Center for Turbulence Research, Stanford (1990), pp. 271-279.

[47] C.J. Rutland and A. Trouvé, Direct simulations of premixed turbulent flames with nonunity lewis numbers, Combust. Flame 94 (1993), pp. 41-57.

[48] D.C. Haworth and T.J. Poinsot, Numerical simulations of lewis number effects in turbulent premixed flames, J. Fluid Mech. 244 (1992), pp. 405-436. 
[49] B. Renou, A. Boukhalfa, D. Puechberty, and M. Trinite, Effects of stretch on the local structure of preely propagating premixed low-turbulent flames with various lewis numbers, Symp. (Int.) Combust. 27 (1998), pp. 841-847.

[50] N. Chakraborty and R.S. Cant, Influence of lewis number on curvature effects in turbulent premixed flame propagation in the thin reaction zones regime, Phys. Fluids 17 (2005), p. 41.

[51] N. Chakraborty and R.S. Cant, Effects of strain rate and curvature on surface density function transport in turbulent premixed flames in the thin reaction zones regime, Phys. Fluids 17 (2005), pp. 1-15.

[52] N. Chakraborty, M. Klein, and R.S. Cant, Effects of turbulent reynolds number on the displacement speed statistics in the thin reaction zones regime of turbulent premixed combustion, J. Combust. 2011 (2011).

[53] W.T. Ashurst, A.R. Kerstein, R.M. Kerr, and C.H. Gibson, Alignment of vorticity and scalar gradient with strain rate in simulated Navier-Stokes turbulence, Phys. Fluids 30 (1987), pp. 2343-2353.

[54] P.J. Diamessis and K.K. Nomura, Interaction of vorticity, rate-of-strain, and scalar gradient in stratified homogeneous sheared turbulence, Phys. Fluids 12 (2000), pp. 1166-1188.

[55] G. Gulitski, M. Kholmyansky, W. Kinzelbach, B. Lüthi, A. Tsinober, and S. Yorish, Velocity and temperature derivatives in high-Reynolds-number turbulent flows in the atmospheric surface layer. part 3. temperature and joint statistics of temperature and velocity derivatives, J. Fluid Mech. 589 (2007), pp. 103-123.

[56] D.A. Donzis and P.K. Yeung, Resolution effects and scaling in numerical simulations of passive scalar mixing in turbulence, Phys. D (Amsterdam, Neth.) 239 (2010), pp. 12781287.

[57] N. Chakraborty and N. Swaminathan, Influence of the Damköhler number on turbulencescalar interaction in premixed flames. I. physical insight, Phys. Fluids 19 (2007), pp. 115-183.

[58] G. Hartung, J. Hult, C.F. Kaminski, J.W. Rogerson, and N. Swaminathan, Effect of heat release on turbulence and scalar-turbulence interaction in premixed combustion, Phys. Fluids 20 (2008), p. 035110.

[59] N. Chakraborty, M. Klein, and N. Swaminathan, Effects of Lewis number on the reactive scalar gradient alignment with local strain rate in turbulent premixed flames, Proc. Combust. Inst. 32 (2009), pp. 1409-1417.

[60] B. Coriton and J.H. Frank, Impact of heat release on strain rate field in turbulent premixed bunsen flames, Proc. Combust. Inst. 36 (2017), pp. 1885-1892. 